\title{
Educação em saúde para idosos com pé diabético hospitalizado em um hospital universitário de Belém: relato de experiência
}

\author{
Health education for elderly with diabetic foot \\ hospitalized in the university hospital of Belém: \\ experience report
}

\author{
Renato da Costa Teixeira', Viviane da Costa Reis ${ }^{2,3}$, José Wagner Cavalcante Muniz ${ }^{3}$ \\ 'Programa de Pós-graduação Profissional em Ensino em Saúde na Amazônia, Universidade do Estado do Pará, \\ Belém (PA), Brasil. \\ 2Prefeitura Municipal de Barcarena, Barcarena (PA), Brasil. \\ ${ }^{3}$ Universidade Federal do Pará, Barcarena (PA), Brasil.
}

Recebido: Dez. 28, 2016 Aceito: Mar. 20, 2017

\section{COMO CITAR ESTE ARTIGO}

Teixeira RC, Reis VC, Muniz JWC Educação em saúde para idosos com pé diabético hospitalizado em um hospital universitário de Belém: relato de experiência. Interdisciplinary Journal of Health Education 2016 Ago-Dez;1(2):132-138. http://dx.doi org/10.4322/ijhe.2016.021

\section{CORRESPONDÊNCIA}

Renato da Costa Teixeira

Programa de Pós-graduação

Profissional em Ensino em Saúde na

Saúde na

Amazônia, Universidade do Estado do

Pará

Travessa Lomas Valentinas, 1354, ap.

501, Marco, CEP 66093-671, Belém (PA), Brasil

Tel.: (91) 3277-3407

teixeirarenato@globo.com

FONTE DE FINANCIAMENTO

O presente estudo foi autofinanciado.

\section{CONFLITO DE INTERESSE}

Os autores declararam não

haver conflitos de interesse.

O estudo foi realizado no Hospital Universitário João de Barros Barreto, localizado no município de Belém (PA), Brasil.

\section{RESUMO}

Introdução: Educação em saúde é uma estratégia preventiva que inclui políticas públicas, ambientes apropriados e reorientação dos serviços de saúde para além dos tratamentos clínicos e curativos, tendo um lugar de importância sobre os determinantes sociais da saúde, uma vez que estas são as condições sociais em que vivem e trabalham as pessoas. Objetivo: $O$ presente artigo tem como objetivo relatar ações educativas em saúde junto a idosos com diagnóstico de pé diabético internados em um hospital universitário de Belém. Método: O estudo foi aprovado pelo comité de ético em pesquisa do hospital e se convidou todos os idosos com pé diabético. A metodologia adotada foi a problematizadora e participativa através de rodas de conversa. Atividades de treinamento e capacitação foram realizadas com a equipe interdisciplinar. Resultados: Participaram das intervenções 6 idosos, com um baixo nível educativo e com renda familiar de até 2 salários mínimos. 0 estudo revelou uma grande satisfação para os participantes, em especial para compartilhar suas historias e enumerar situações em relação ao processo saúde-doença no grupo, alguns mostraram duvidas quanto ao conhecimento dos fatores relacionados a causa, o tratamento não farmacológico e o autocuidado. Por outro lado, demostraram o domínio da informação relacionada aos fatores predisponentes, a natureza crônica da enfermidade e as complicações. Considerações: Esta experiência teve como limitações a falta de espaço e recursos, a disponibilidade de horários que no supõe um prejuízo à rotina do paciente e resistência por parte de alguns profissionais assistentes. Por fim, este tipo de atividade educativa deve ser estimulado entre os profissionais de saúde, assim como a promoção da assistência de integração para promover o envelhecimento saudável com as pessoas afetadas por enfermidades crônicas.

Palavras-chave: Educação em saúde. Pé diabético. Saúde do idoso.

\section{ABSTRACT}

Introduction: Health education is a preventive strategy that includes public policies, appropriate environments and reorientation of health services beyond clinical and curative treatments, taking an important place on the social determinants of health, since these are the social conditions in which people live and work. Objective: This is an experience report, whose aim is to present educational activities in health in the elderly with diabetic foot diagnosis admitted to a university hospital in Belém. There were conducted classes and educational activities in an interdisciplinary team. Methods: The study was approved by the Ethics Committee of hospital. They were invited to attend all elderly diabetics hospitalized for diabetic foot problems. Results: The methodology was the discussion of problems and active participation. Participated in interventions six seniors, with little schooling and family income of up to two minimum wages. Revealed a great satisfaction from the participants, especially to tell their stories to the group and list specific situations as their life and health, they demonstrated some uncertainty as to the knowledge of factors related to the cause, non-pharmacological treatment and self-care. However, the elderly demonstrated to know enough about information related to predisposing factors, chronic nature of the disease and complications. Considerations: This experience had difficulties as the lack of space and resources, the availability of schedules that would not undermine the patient's daily activities and resistance by some assistants professionals. Finally, such educational activities need to be encouraged, promoting integration in between assistance to promote healthy aging with the people affected by chronic diseases.

KEYWORDS: Health education. Diabetic foot. Health of the elderly. 


\section{Contextualização}

O diabetes Mellitus (DM) é considerado um dos mais importantes problemas de saúde pública, é responsável por grande número de hospitalizações prolongadas e onerosas, absenteísmo, aposentadoria precoce e redução da qualidade de vida dos indivíduos devido suas complicações devastadoras. Dentre essas complicações destaca-se o pé diabético, pois pode evoluir para ulcerações e amputações ${ }^{1}$.

Segundo a pesquisa Vigilância de Fatores de Risco e Proteção para Doenças Crônicas por Inquérito Telefônico ${ }^{2}$, o percentual de pessoas que se declararam diabéticas passou de $5,3 \%$ para 7,4\%, entre 2006 e 2012. Este estudo destacou a educação como um importante fator de prevenção: 3,8\% dos brasileiros com mais de 12 anos de estudo declararam ser diabético, enquanto que este percentual é muito maior entre aqueles que tem somente até 8 anos de estudo (12,1\%).

A Pesquisa Nacional de Saúde, realizada pelo Ministério da Saúde em parceria com o IBGE ${ }^{3}$, mostra que a diabetes afeta cerca de 9.000 .000 brasileiros e expõe os percentuais de prevalência da doença por faixa etária, que são: $0.6 \%$ entre 18 e 29 anos de idade; $5 \%$ entre 30 a 59 anos; $14.5 \%$ entre 60 e 64 anos; $19,9 \%$ entre 65 e 74 anos e $19.6 \%$ para maiores de 75 anos o mais de idade, mostrando que a prevalência é maior em populações maiores.

Considerando o cenário de envelhecimento populacional, mudanças nos padrões de consumo, nos estilos de vida e urbanização, há consequente aumento das condições de doenças crônicas. Então, ações de educação em saúde configuram-se como uma estratégia primordial para o controle da doença, prevenção de agravos e atenção às necessidades desse grupo social ${ }^{4}$.

Na população idosa esses acometimentos são mais preocupantes, de modo que as políticas públicas de saúde devem favorecer um envelhecimento saudável, reduzindo o quadro de incapacidade funcional e evitando os altos índices de complicações e hospitalizações ${ }^{5,6}$.

As Diretrizes da Sociedade Brasileira de Diabetes ${ }^{7}$ preconizam que a educação em diabetes deve ser parte integrante do cuidado com o paciente. A cronicidade da doença requer acompanhamento contínuo ao indivíduo, portanto a educação em saúde deveria ser um processo permanente, gradativo e interativo, utilizando estratégias de abordagem individual e em grupo, em qualquer local em que se encontra o indivíduo.

O acesso à educação é fundamental para que os indivíduos possam controlar sua saúde, tomar decisões e adotar hábitos saudáveis. Desse modo, a educação em saúde é uma estratégia que possibilita esse empoderamento do usuário, pois permite que ele adquira conhecimento de si mesmo e dos determinantes sociais que fazem parte da sua vida, podendo exercer mudanças no seu ambiente e na sua própria conduta ${ }^{8,9}$.

Tais intervenções são raramente realizadas no âmbito hospitalar ${ }^{10}$, deixando os usuários em atenção terciária a margem do processo educativo em saúde. Tendo em vista essa conjuntura, o presente estudo se propõe a relatar experiência de ação educativa em saúde junto a idosos com pé diabético internados em um hospital universitário de Belém.

\section{Descrição da experiência}

Trata-se de um relato de experiência de uma ação educativa em saúde, realizada na Clínica Médica do Hospital Universitário João de Barros Barreto, o qual é um hospital de referência na região Norte e assiste indivíduos oriundos tanto da região metropolitana de Belém quanto de municípios circunjacentes. O estudo foi aprovado pelo Comitê de Ética em Pesquisas do HUJBB através do Parecer n ${ }^{\circ}$ 1.395.529.

Todos os idosos hospitalizados com diagnóstico de pé diabético foram convidados pessoalmente pela equipe composta por uma Fisioterapeuta, duas Terapeutas Ocupacionais e uma Psicóloga. Devido à abordagem exigir entendimento, 
compreensão e discussão das temáticas propostas, aqueles que apresentavam déficit cognitivo (avaliado pelo Miniexame do Estado Mental) foram excluídos das atividades.

A ação foi composta por cinco etapas: a) treinamento da equipe de trabalho; b) avaliação cognitiva e coleta de dados dos participantes; c) duas ações educativas; d) avaliação da percepção dos participantes; e) avaliação e percepção da equipe de trabalho. Todas as atividades junto aos idosos foram executadas na própria enfermaria de internação do idoso, enquanto o treinamento e avaliação da equipe foram realizados no centro de estudo do hospital.

O treinamento da equipe foi realizado nos meses de outubro e novembro de 2015, as intervenções junto aos idosos e avaliação das ações ocorreram em dezembro de 2015 e janeiro de 2016.

Essas atividades foram estabelecidas a partir da metodologia problematizadora e participativa, que tem como fundamentação a atuação efetiva dos participantes no processo educativo sem considerá-los meros receptores, baseada na percepção da realidade, protagonismo e trabalho em grupo $^{11}$.

Tais atividades tiveram como objetivo compartilhar e debater informações sobre Diabetes mellitus, buscando identificar conhecimentos prévios, limitações, necessidades, percepção sobre o processo saúde-doença da Diabetes mellitus. Foram abordados aspectos referentes à definição, fatores predisponentes, sintomas, complicações, tratamento, implicações socioculturais, sobretudo, relativas aos hábitos alimentares e prática de atividades físicas, a prevenção terciária, controle e autocuidado, especialmente o cuidado com os pés.

Os pontos para reflexão, debate e propostas de dinâmicas de grupo se basearam na Cartilha de Promoção da Saúde e Envelhecimento da Universidade Aberta da Terceira Idade ${ }^{12}$, sendo adotada linguagem adequada ao público leigo e recursos diferenciados, como imagens, cartazes, questões norteadoras, relatos de experiências e jogos levados pelos facilitadores ou construídos pelos participantes.

Para construção das intervenções educativas juntos aos idosos hospitalizados, equipe foi capacitada em encontros semanais, nos quais foram abordados conteúdos referentes à Diabetes mellitus, seus determinantes sociais e repercussão no processo saúde-doença, promoção, prevenção, educação em saúde, trabalho interdisciplinar, procedimentos de avaliação e metodologia de intervenção com metodologias de aprendizagem ativa para este fim. Quatro profissionais de saúde e cinco acadêmicos participaram desta etapa, porém somente os professionais e saúde se mantiveram em todas as etapas da atividade educativa com os idosos, já que os acadêmicos não demostraram ter tempo para as atividades.

A segunda etapa consistiu na avaliação cognitiva através do Miniexame do Estado Mental, eleição de participantes e aplicação de questionários individualmente, com o objetivo de levantar informações que possibilitassem caracterização do grupo, a fim de conhecer os indivíduos quanto ao gênero, idade, renda e escolaridade, pois essas particularidades interferem diretamente no processo de cuidados em saúde.

Foram avaliados onze idosos, porém somente seis tinham nível cognitivo compatível com as atividades e foram convidados a participar na atividade educativa. A maioria composta por homens, entre 63 e 75 anos, de baixa escolaridade (ensino fundamental incompleto) e com renda familiar de até 2 salários mínimos.

$\mathrm{Na}$ terceira etapa, estes idosos foram reunidos em duplas. Iniciou-se com a apresentação de todos os sujeitos envolvidos (equipe da saúde e participantes) e, por meio de questões norteadoras, cada um relatava sua história, experiências, percepções e conhecimentos quanto a Diabetes mellitus.

Após este momento, a equipe buscava identificar as principais dificuldades dos idosos quanto à compreensão de fatores relacionados ao processo saúde-doença do Diabetes mellitus e, a partir disso, abordar tais elementos na intervenção subsequente, que consistia em dinâmica de julgar e comentar itens verdadeiros e falsos, descrever 
as imagens apresentadas (Figura 1) que ilustravam cuidados com os pés, explanar sobre a relação do que era discutido com suas experiências pessoais.

Durante essa atividade foi enfatizado o autocuidado com os pés, resultando na troca de conhecimento e de interação entre os idosos e profissionais (Figura 2).

No último encontro, os idosos relataram suas impressões pessoais quanto às ações realizadas, os pontos positivos e negativos e qual a importância destas para seus cuidados de saúde.

Esse conjunto de ações teve como objetivo auxiliar no processo de autonomia destes pacientes, sensibilizando-os e estimulando o conhecimento e reflexão sobre a saúde.

Por fim, a equipe realizou reunião de avaliação e discussão sobre as atividades realizadas.

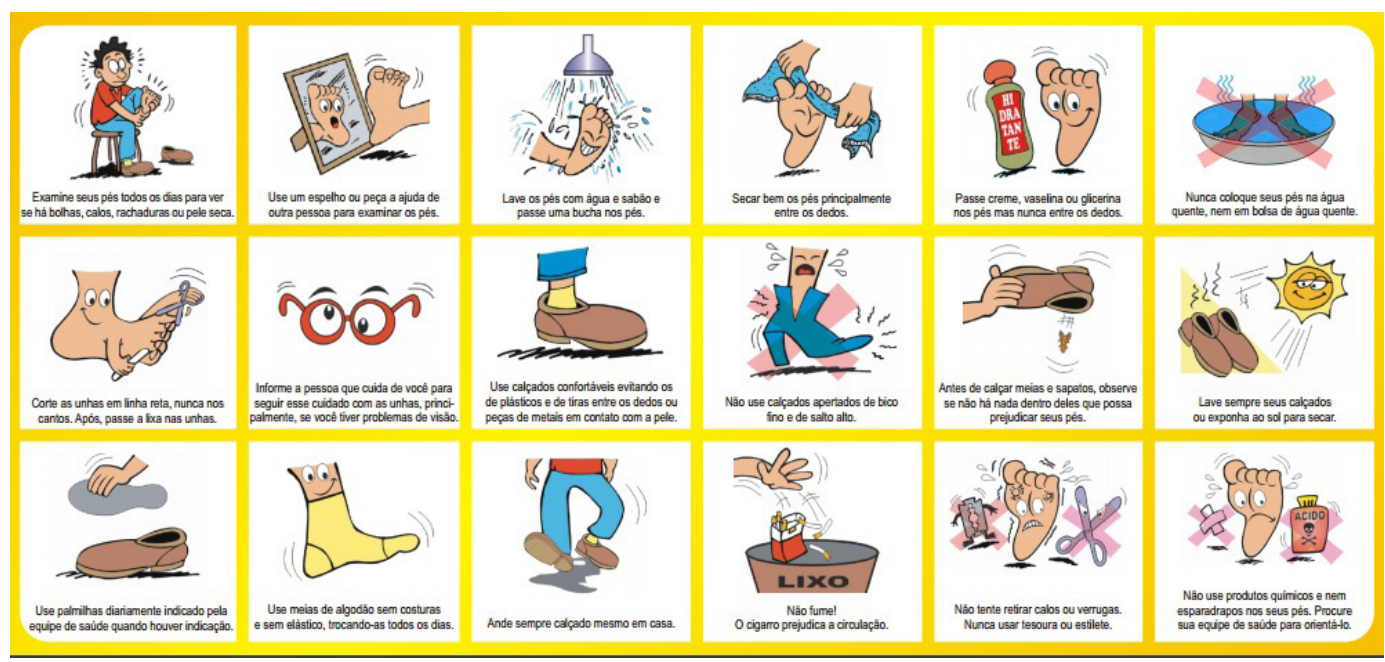

Figura 1. Imagens utilizadas na ação educativa. Fonte: Bahia13.

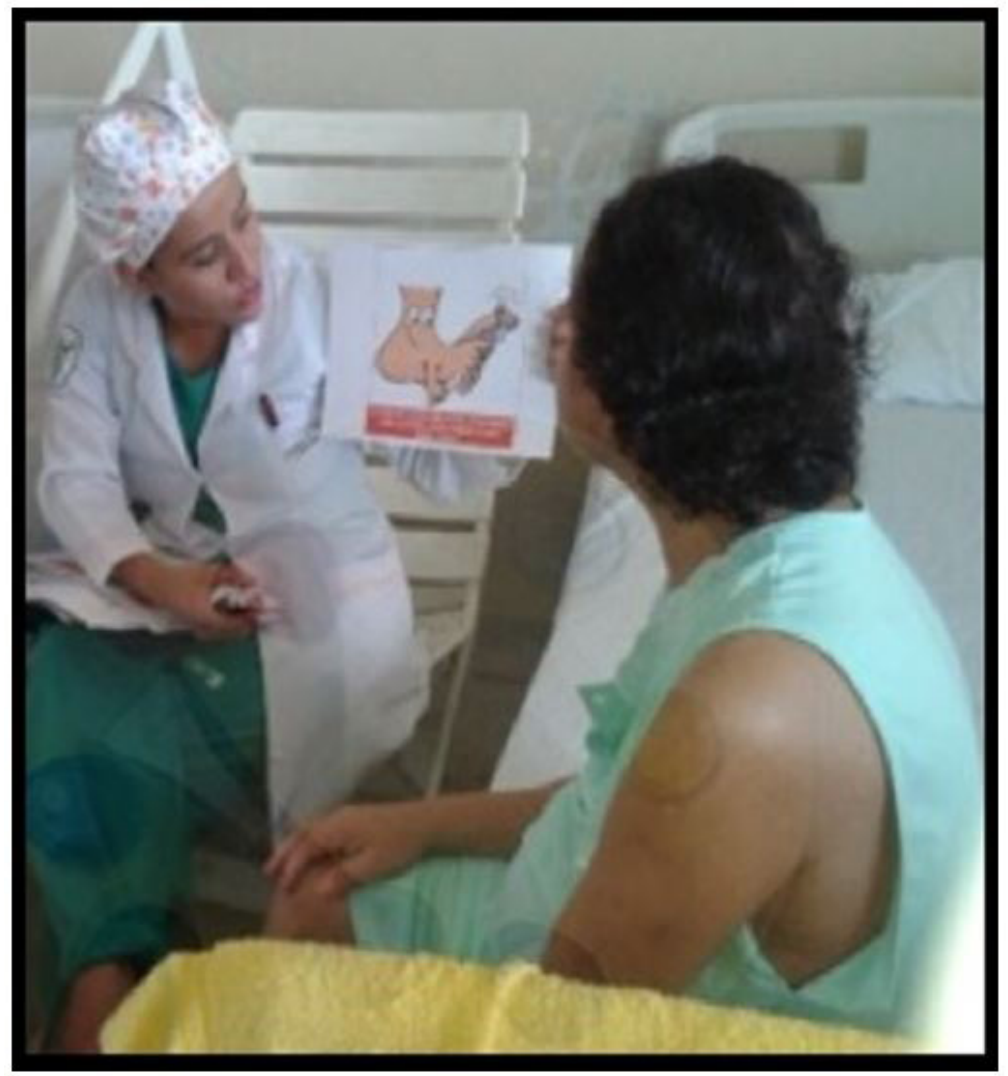

Figura 2. Profissional conversando sobre o cuidado com os pés. Fonte: Arquivo pessoal. 


\section{Resultados e impactos}

Diante do modelo de atenção à saúde predominantemente voltando para o biologicismo, com ações restritas ao tratamento sintomático e focado na doença, trabalhar com educação em saúde é um grande desafio, em especial quando está voltada para alguma doença crônica no âmbito hospitalar ${ }^{4}$.

Segundo Pereira ${ }^{14}$, podemos entender a educação em saúde como formas do homem reunir e dispor recursos para intervir e transformar as condições objetivas, visando alcançar a saúde como um direito socialmente conquistado, a partir da atuação individual e coletiva de sujeitos político-sociais.

Portanto, é um processo participativo de afirmação de sujeitos e construção de cidadania. Para seu sucesso, deve ser composta por: planejamento, execução e avaliação ${ }^{12}$. Tais etapas foram empregadas na experiência relata. Bezerra et al. ${ }^{15}$ descrevem que há necessidade de estimular os profissionais de saúde a reflexão sobre o seu trabalho e atualização sobre as diversas formas de intervenção e cuidados de saúde.

Estudo sobre a prática educativa do enfermeiro com o paciente e com a família expõe que estas ações acontecem raramente no âmbito hospitalar e, quando realizadas, são nos momentos de admissão ou alta. Além disso, identificou presença de forte tendência da forma tradicional de transmissão do conhecimento ${ }^{10}$.

Nesse sentido, as ações de educação em saúde para idosos necessitam de metodologias que considerem a complexidade do processo de envelhecimento, tal qual foi conduzida nesta experiência, e relacionem os fatores que permeiam a vida do indivíduo, como as crenças, cultura, estilos de vida e aspectos psicossociais. Afinal, somente levando em consideração os conhecimentos, a cultura e o meio em que vivem os idosos é que se obterão os resultados almejados ${ }^{9,16}$.

Desse modo, as estratégias precisam ser adequadas e o conteúdo deve ser compartilhado (e não somente transmitido) de forma simples, respeitosa e motivadora, visando que o indivíduo compreenda a doença e assuma, de forma ativa, seu papel no tratamento e nos cuidados ${ }^{12}$. Nessa experiência, os idosos, assim como os cuidadores, demonstraram-se receptivos, participativos e interessados na execução das atividades.

Evidenciou-se grande satisfação por partes dos participantes, especialmente ao partilhar suas histórias e enumerar situações particulares quanto ao processo saúdedoença em grupo. O grupo educativo pode ser considerado um espaço de crescimento, vínculo entre participantes e melhoria da relação terapeuta-paciente ${ }^{17}$.

Embora os idosos convivam com a doença há muitos anos, demonstraram insegurança quanto ao conhecimento de fatores relacionados à causa, tratamento não farmacológico e autocuidado. Estudo de Oliveira e Zanetti ${ }^{18}$, em pesquisa realizada com 79 idosos, também encontraram valores insatisfatórios quanto ao conhecimento sobre autocuidado. A dificuldade quanto ao conhecimento sobre causa e formas de tratamento não farmacológico pode estar relacionada com a baixa escolaridade.

Por outro lado, os idosos têm domínio sobre aspectos relacionados aos fatores predisponentes, caráter crônico da doença e complicações. Porém, é muito difícil mudar comportamentos de risco sem transformar as normais culturais que os influenciam ${ }^{19}$.

Por isso, é importante investir no fortalecimento, organização e participação das pessoas na comunidade. Ações coletivas podem favorecer a melhoria das condições de saúde e bem estar ${ }^{19}$.

A limitação no processo de implementar novas ações, baseadas nos princípios da educação em saúde, autores, como Moutinho et al. ${ }^{20}$, citam a pouca disponibilidade de recursos materiais e estruturais e desarticulação da equipe de saúde. Na experiência relatada, foram observadas essas restrições, além de outras como a resistência de alguns profissionais da saúde e dificuldade para disponibilidade de horários que não prejudicassem a rotina do paciente no hospital. 
Entretanto, tais problemas puderam ser contornados pela boa relação da equipe de trabalho com os profissionais assistentes e adequada organização das abordagens.

Por fim, ressaltamos que essa experiência foi de grande valor para uma discreta mudança no modelo de assistência prestado aos pacientes hospitalizados, assim como contribuiu para o conhecimento e experiência interdisciplinar da equipe de trabalho e para conhecimento, autonomia e bem-estar dos participantes.

\section{Considerações finais}

A prática de educação em saúde deveria estar contemplada em todos os níveis de atenção à saúde, porém ainda há carência de tais ações nos cuidados hospitalares. O profissional de saúde ainda não se enxerga como agente educador e corresponsável pela transformação de hábitos e como auxiliar no desenvolvimento de autonomia e empoderamento dos indivíduos. Nessa perspectiva, atividades educativas como esta precisam ser incentivadas, promovendo integração da assistência à promoção do envelhecimento saudável, mesmos junto a indivíduos acometidos por doenças crônicas. Portanto, espera-se que novas abordagens sejam realizadas nesse sentido.

\section{Referências}

1. Vilar L. Endocrinologia clínica. Rio de Janeiro: Guanabara Koogan; 2013.

2. Brasil. Ministério da Saúde. Vigitel Brasil 2012: vigilância de fatores de risco e proteção para doenças crônicas por inquérito telefônico [Internet]. Brasília; 2012 [citado em 2016 Jun 5]. Disponível em: http://bvsms.saude.gov.br/bvs/publicacoes/ vigitel_brasil_2012_vigilancia_risco.pdf

3. Instituto Brasileiro de Geografia e Estatística - IBGE. Um panorama da saúde no Brasil: acesso e utilizacão de serviços, condicões de saúde e fatores de risco e proteção à saúde, 2008: pesquisa nacional por amostra de domicílios [Internet]. Rio de Janeiro; 2008 [citado em 2016 Jun 2]. Disponível em: http://bvsms.saude.gov.br/bvs/publicacoes/pnad_panorama_saude_brasil. pdf

4. Mendes EV. As redes de atenção à saúde. Brasília: Organização Pan-Americana da Saúde; 2011.

5. Veras R. Envelhecimento populacional contemporâneo: demandas, desafios e inovações. Rev Saude Publica. 2009;43(3):54854. PMid:19377752. http://dx.doi.org/10.1590/S0034-89102009000300020.

6. Minayo MCS. O envelhecimento da população brasileira e os desafios para o setor saúde. Cad Saude Publica. 2012;28(2):209.

7. Sociedade Brasileira de Diabetes - SBD. Diretrizes da SBD: 2014-2015. São Paulo: AC Farmacêutica; 2015.

8. Torres HC, Franco LJ, Stradioto MA, Hortale VA, Schall VT. Avaliação estratégica de educação em grupo e individual no programa educativo em diabetes. Rev Saude Publica. 2009;43(2):291-8. PMid:19225700. http://dx.doi.org/10.1590/ S0034-89102009005000001.

9. Geib LT. Determinantes sociais da saúde do idoso. Cien Saude Colet. 2012;17(1):123-33. PMid:22218546. http://dx.doi. org/10.1590/S1413-81232012000100015.

10. Rigon AG, Neves ET. Educação em saúde e a atuação de enfermagem no contexto de unidades de internação hospitalar: o que tem sido ou há para ser dito? Texto Contexto Enferm. 2011;20(4):812-7. http://dx.doi.org/10.1590/S0104-07072011000400022.

11. Freire P. Pedagogia da autonomia: saberes necessários à prática educativa. 21. ed. São Paulo: Editora Paz e Terra; 2015.

12. Assis M. Promoção da saúde e envelhecimento: orientações para o desenvolvimento de ações educativas com idosos. Rio de Janeiro: UnATI: 2002.

13. Bahia. Governo do Estado. O pé do diabético: o diabético tem um bom motivo para cuidar dos pés [Internet]. Salvador; 2016 [citado em 2016 Jun 2]. Disponível em: http://www.saude.ba.gov.br/cedeba/cartaz_pe_diabetico.pdf

14. Pereira IB. Dicionário da educação profissional em saúde. 2. ed. Rio de Janeiro: EPSJV; 2008.

15. Bezerra IMP, Oliveira AKS, Silva CC, Lima EA No, Silva ATMC. Velhas e novas formas de intervenção em saúde: os modelos assistenciais em análise. Saúde em Debate. 2011;35(89):197-206.

16. Mallmann DG, Galindo NM No, Sousa JC, Vasconcelos EMR. Educação em saúde como principal alternativa para promover a saúde do idoso. Cien Saude Colet. 2015;20(6):1763-72. PMid:26060954. http://dx.doi.org/10.1590/1413-81232015206.02382014.

17. Caires MG, Araújo A. Grupo educativo com adolescentes diabéticos: um relato de experiência. Adolescente e Saude. 2013;10(1):56-62.

18. Oliveira KC, Zanetti ML. Conhecimento e atitude de usuários com diabetes mellitus em um Serviço de Atenção Básica à Saúde. Rev Esc Enferm USP. 2011;45(4):862-8. PMid:21876885. http://dx.doi.org/10.1590/S0080-62342011000400010.

19. Buss PM, Pellegrini A Fo. A saúde e seus determinantes sociais. Physis. 2007;17(1):77-93. http://dx.doi.org/10.1590/ S0103-73312007000100006.

20. Moutinho CB, Almeida ER, Leite MTS, Vieira MA. Dificuldades, desafios e superações sobre educação em saúde na visão de enfermeiros de saúde da família. Trabalho Educação e Saúde. 2014;12(2):253-72. 


\section{ijhe}

\section{Contribuição dos autores}

Todos os autores, Renato da Costa Teixeira, Viviane da Costa Reis, José Wagner Cavalcante Muniz participaram da elaboração e execução do projeto que gerou o presente relato e da elaboração do manuscrito de forma total, assumindo a responsabilidade completa pelo conteúdo do mesmo, tendo ambos participado da redação e revisão crítica do conteúdo. 